\title{
EVALUATION OF ANISOTROPIC FILTERS FOR DIFFUSION TENSOR IMAGING
}

\author{
Jee Eun Lee ${ }^{1,2}$, Moo K. Chung ${ }^{1,3}$, Andrew L. Alexander ${ }^{1,2,4}$ \\ Waisman Laboratory for Functional Brain Imaging and Behavior ${ }^{1}$, \\ Departments of Medical Physics ${ }^{2}$, Statistics ${ }^{3}$, Psychiatry ${ }^{4}$, \\ University of Wisconsin, Madison, WI
}

\begin{abstract}
Diffusion tensor imaging (DTI) measures, such as fractional anisotropy (FA), and trace are very sensitive to noise contained in the acquired diffusion weighted images. Typical isotropic smoothing methods reduce the high spatial frequency image content and blur the image features. We hypothesized that the diffusion tensor would be an approximate anisotropic Gaussian filter function because the blur will tend to be oriented parallel to the white matter structures. Thus, we implemented and evaluated an anisotropic Gaussian kernel smoothing method based on the diffusion tensor for preserving diffusion tensor structural features while significantly reducing the noise. We compared the diffusion tensor anisotropic filtering with isotropic Gaussian filtering, and a Perona-Malik (PM) filtering algorithm, which was derived from the intensity gradients of diffusion weighted images. Human brain DTI data with high SNR was used as a gold standard for evaluation. Overall, the anisotropic filters performed similarly, with slightly better performance using the DT anisotropic filter across the whole brain.
\end{abstract}

\section{INTRODUCTION}

Diffusion tensor MRI (DT-MRI) is a non-invasive imaging method for assessing the characteristics of tissue microstructure. The number and breadth of DT-MRI applications are rapidly expanding. Unfortunately, diffusion tensor measurements are also highly sensitive to noise in the raw images [6] and, the variance of DT-MRI measures may impair the ability to detect and characterize subtle differences between regions or subjects.

Image filtering and smoothing methods may be used to reduce noise in medical images. However, certain types of smoothing may also blur important image features and the edges of structures.
Fine image features and edges may be preserved using anisotropic diffusion filtering methods such as Perona-Malik (PM) algorithm [5]. PM filter was originally developed for scalar images and methods for smoothing DT-MRI data may require more complex approaches than scalar image smoothing methods, because the diffusion tensor image data is multidimensional and represents spatially coherent directional information by the eigenvectors and eigenvalues. Recently, several studies have investigated methods for filtering DT-MRI data. Parker et al. [4] applied PM algorithm to the raw, diffusion-weighted scalar images prior to calculation of the diffusion tensor and associated measures. Pajevic et al. used a B-spline interpolation method to regularize the diffusion tensor field [6]. More recently, several investigators have applied constrained variational principles to the full diffusion tensor data with promising results (Tschumperle et al. [7]; Coulon et al. [2]; Wang et al. [8]). However, these approaches have not been widely used because they are relatively complex and the computational demands can be high. Chung et al. proposed using the diffusion tensor at each voxel as the anisotropic diffusion kernel [1]. The application of the diffusion tensor as the filter kernel will inherently smooth the data more in the direction of greatest diffusivity, which is generally parallel to the orientation of white matter tracts in the brain. Conversely, in gray matter areas, which demonstrate more isotropic diffusion, the smoothing will also be more isotropic.

In this paper, anisotropic kernel smoothing approaches similar to those described by Chung et al. are compared against isotropic Gaussian smoothing and the Perona Malik filtering algorithm. The performance of this spatial filtering method is evaluated using a root mean square error (RMSE) that represents the accuracy and variance of diffusion tensor measures. DTI measurements in a human brain are used to evaluate the filter performance. Finally, several implementations of the DTI anisotropic filter are evaluated. 


\section{THEORY}

\subsection{Gaussian Kernel Smoothing}

A single 3D image smoothing operation may be described using the convolution

$$
S_{i+1}=K_{t} \otimes S_{i} \quad \text { [Equation 1] }
$$

where $S_{i}$ and $S_{i+1}$ are the signals for the ith and $(\mathrm{i}+1)$ th iterations ( $S_{0}$ is the original unfiltered image) respectively, and $\mathrm{K}_{\mathrm{t}}$ is the convolution kernel.

The subscript $t$ is called diffusion time or the bandwidth of the kernel. The effective convolution kernel size may be increased by repeated convolution

$$
K_{\sqrt{n t}}=K_{t} \otimes K_{t} \otimes K_{t} \ldots \otimes K_{t} \quad \text { (n times) } \quad \text { [Equation 2] }
$$

For many image processing applications, an isotropic Gaussian kernel is used for smoothing

$$
K(\vec{r})=\frac{\exp \left(-|r|^{2} / 2 \sigma^{2}\right)}{(2 \pi)^{3 / 2} \sigma^{3}}
$$

where $\vec{r}$ is the position vector, and $c$ is the standard deviation of the distribution $K(\vec{r})$ in the three dimensional case. However, isotropic filters are completely non-specific and will blur signals between neighboring voxels regardless of the local tissue organization. The diffusion tensor is a model of Gaussian diffusion and the major orientation in white matter is parallel to the local tract organization. Consequently, the diffusion tensor, $\overline{\mathrm{D}}$, may be used to create a more tissue specific smoothing kernel

$$
K_{t}(\vec{r})=\frac{\exp \left(-r^{T} \overline{\mathrm{D}}^{-1} r / 4 t\right)}{(4 \pi t)^{3 / 2}(\operatorname{det} \overline{\mathrm{D}})^{1 / 2}} \quad \text { [Equation 4] }
$$

for convolution image smoothing. This is a solution to the diffusion equation in the three-dimensional at diffusion time $t$.

The anisotropy of the diffusion tensor may be accentuated by raising the diffusion tensor to a higher power, $\overline{\mathrm{D}}^{P}$.

$$
K_{t}(r)=\frac{\exp \left(-\mathrm{r}\left(\overline{\mathrm{D}}^{P}\right)^{-1} \mathrm{r} / 4 t\right)}{(4 \pi t)^{n / 2}\left(\operatorname{det}\left(\overline{\mathrm{D}}^{P}\right)\right)^{1 / 2}}
$$

[Equation 5]

This is achieved by multiplying $\overline{\mathrm{D}}$ by itself $\mathrm{p}$ times. Figure 1 demonstrates how diffusion tensor based Gaussian kernel represents anisotropic profile as opposed to the isotropic Gaussian profile. In this study, $\mathrm{p}=0$ (isotropic), and 3 were tested.

\subsection{Perona Malik Algorithm}

The Perona Malik algorithm is based on the diffusion equation

$$
\frac{\partial I}{\partial t}=\operatorname{div}[g(\|\nabla I\|) \nabla I] \quad \text { [Equation 6] }
$$

, where $\|\nabla I\|$ is the image intensity gradient and $\mathrm{g}$ is a diffusion function that should be in inverse relationship with $\|\nabla I\|$ such as

$g(\|\nabla I\|)=\exp \left[(-\|\nabla I\| / K)^{2}\right] \quad$ [Equation 7]

from Parker et al [4].
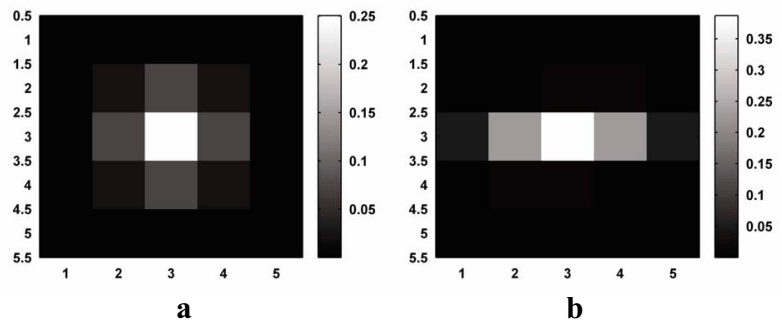

Figure 1. Example of smoothing kernels (x-y plane only) for a voxel in the corpus callosum for (a) isotropic Gaussian smoothing i.e. $p=0$, and (b) $p=3$ anisotropic kernels. The anisotropic kernels show increased preferential smoothing in the $\mathrm{x}$ direction, which is parallel to the WM structure of the corpus callosum.

\section{METHODS}

\subsection{DTI Acquisition}

A single-shot spin echo EPI sequence with diffusion-tensor encoding (12 directions, $b=1000 \mathrm{~s} / \mathrm{mm}^{2}$ ), was used to get four sets (identical slice locations, voxels $=0.8984 \times 0.8984$ $\mathrm{x} 1.8 \mathrm{~mm}^{3}$, 54 slices, $3 \mathrm{NEX}, 23 \mathrm{~cm}$ FOV) DTI data from a single subject. "Gold standard" FA images were estimated by averaging all four sets of diffusion weighted images.

\subsection{Evaluation of Anisotropic Smoothing}

Isotropic, anisotropic Gaussian smoothing kernel with power 3 [Equation 5] and PM filter, were applied independently to each of the four data sets. Various kernel widths were investigated by using the iterative convolution in [Equation 2] up to 10 times with a range of initial t-value $[0.05,2.0]$, and we found the optimum t- value for each filter, $t=0.8$ for Gaussian filter, and $t=0.2$ for PM filter. 
Each optimum filter reaches the minimum RMSE. Filtering was applied to the diffusion-weighted (DW) maps.

The effects of these image filters on maps of FA were evaluated. The error at each voxel for each iteration, $i$, was quantified as the root mean square error between the gold standard maps, $\tilde{x}$, and the smoothed data, $\hat{x}_{i}$,

$$
R M S E_{i}=\sqrt{<\Sigma\left(\tilde{x}-\hat{x}_{i}\right)^{2}>}
$$

[Equation 8]

In this study, the effects of filtering on maps of fractional anisotropy measures were evaluated. The measures were computed for the entire brain volume within the images. Specific regions both within relatively homogenous tissue areas (WM) and areas at tissue interfaces (e.g., GM/WM) were also investigated. Regions of CSF were excluded from the analysis by using a trace threshold (trace $>0.006 \mathrm{~mm}^{2} / \mathrm{s}$ )

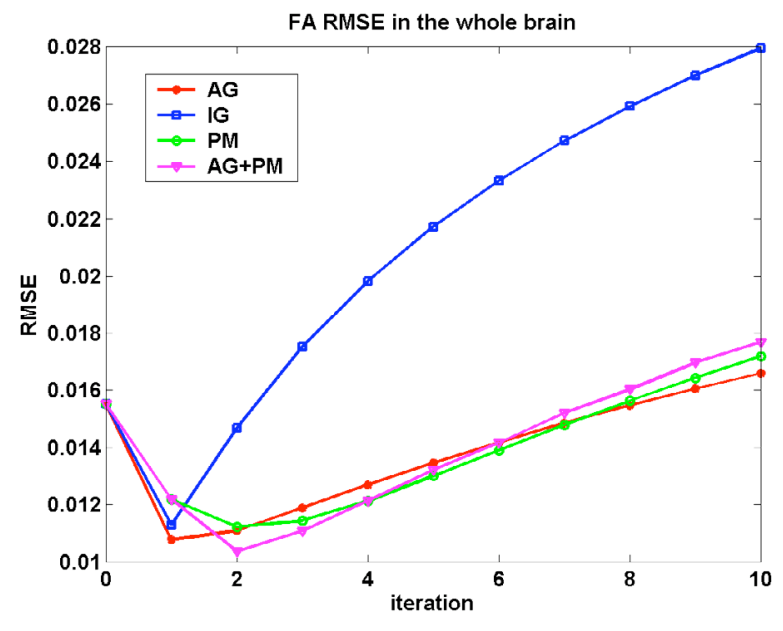

Figure 2. The effects of filtering on FA in the whole brain region. The plots show the effect of different filters on RMSE (Equation 8).

\section{RESULTS}

\subsection{Filtered Image Analysis}

The estimated RMSE values (Equation 8) for all filters are plotted in Figure 2 for FA over the whole brain. In all cases, the error increased after several iterations because of partial volume averaging. Isotropic Gaussian (IG) filtering dramatically increased the error after the first iteration, especially in white matter regions with high anisotropy (FA $>0.4$, data not shown), and performed very well in the gray matter tissue regions with relatively low anisotropy (FA $<0.15)$. Both anisotropic filters (AG and PM) demonstrated less error with large numbers of iterations. A combined $\mathrm{PM}$ and $\mathrm{AG}$ filter (PM+AG in Figure 2), which was designed to apply the PM and AG filter alternately at each iteration, was also evaluated. The combined filter demonstrated the overall minimum error and the resultant smoothed FA maps appear the sharpest [Figure 4].

\subsection{ROI-Specific Analyses}

The filter performance was also evaluated in specific brain regions to examine the filter behavior with the local tissue environment. ROI 1 was placed in a boundary area of between GM and WM. ROI 2 was placed in a highly anisotropic region in the body of the corpus callosum, which was assumed to be relatively homogeneous and parallel. ROI 4 was placed in the globus pallidus, which is considered to be a region of relatively homogenous GM.

ROI 1 - WM/GM Boundary. At tissue interfaces, we expect filtering to be less effective and increase error. Isotropic Gaussian filter increased the error very rapidly, whereas the PM filter was more effective in minimizing partial voluming errors than the anisotropic Gaussian filter.

ROI 2 - Corpus Callosum. The anisotropic Gaussian filter introduced very little error into the FA estimates of homogenous WM [Figure 3].

ROI 3 - Globus Pallidus Gray Matter. In homogenous GM, the anisotropic and isotropic Gaussian filters were nearly equivalent in reducing the RMSE. The PM filter slowly reduced the RMSE since the $t$-value was small, $t=0.2$.

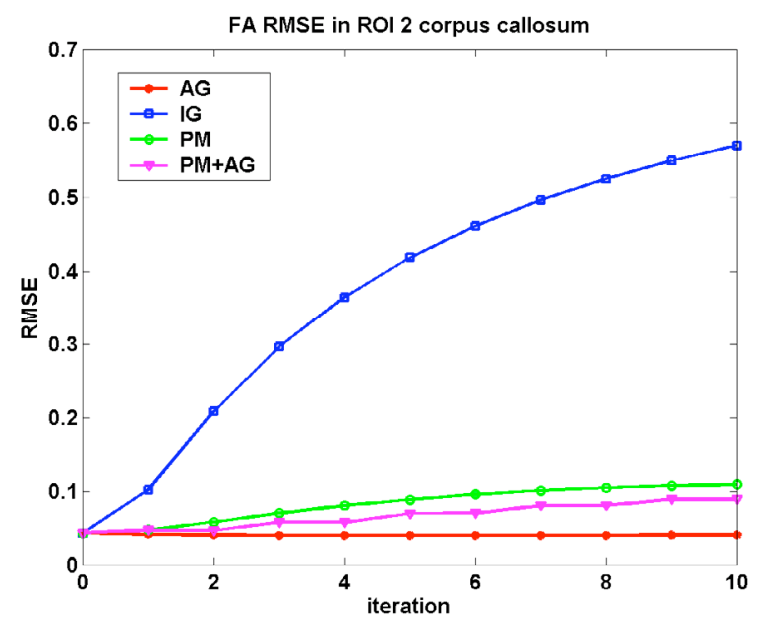

Figure 3. The effects of filtering on FA in corpus callosum.

\subsection{Visualization of Maps from Filtered Data}

The effects of filtering on DTI maps after two iterations are displayed in Figure 4. The spatial texture of the FA maps is 
clearly smoother for all filtering methods. However, the features in the FA maps are sharper with anisotropic filters. The error in FA, particularly in WM regions such as the corpus callosum, is reduced. The minimum RMSE that was achieved by filtering and visual inspection suggest that the combination of the PM and the Anisotropic Gaussian filter was the most effective filtering method in our evaluation.

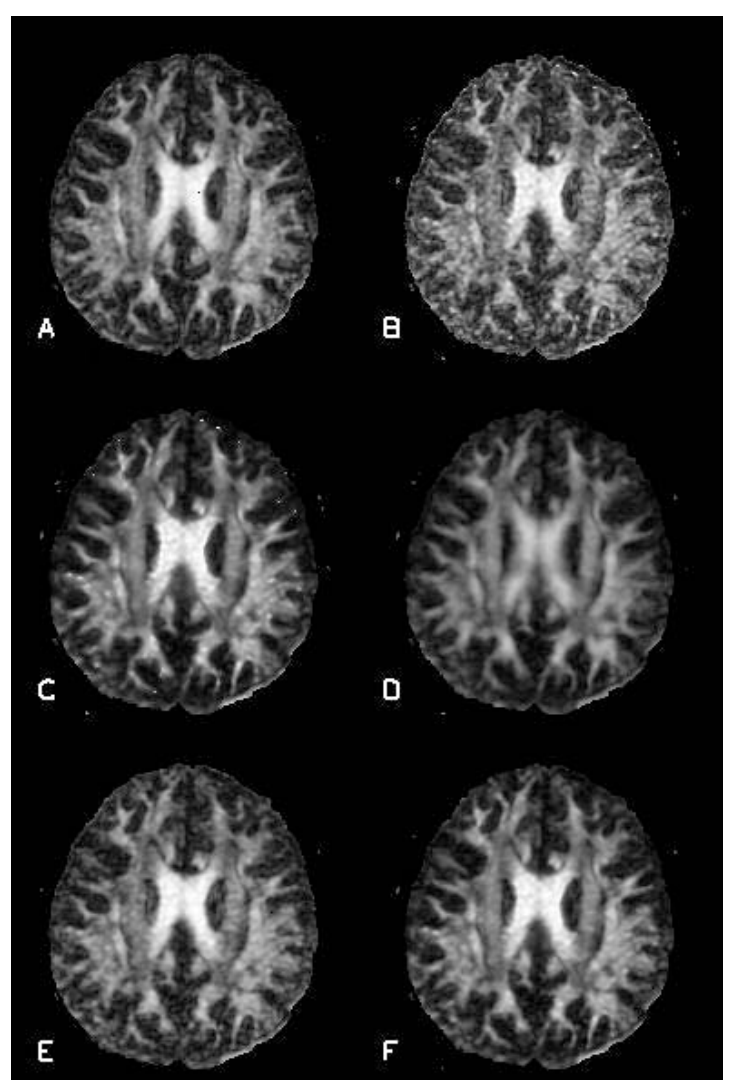

Figure 4 (A) Gold standard, (B) unfiltered, (C) AG filtered, (D) IG filtered, (E) PM filtered, (F) PM+AG filtered FA maps at the $2^{\text {nd }}$ iteration in Figure 2

\section{CONCLUSIONS}

In this study, anisotropic smoothing kernels based upon the diffusion tensor, an isotropic Gaussian distribution and the Perona Malik algorithm were investigated for reducing noise in diffusion tensor image data. We had hypothesized that anisotropic kernels would effectively reduce noise in DTI data with less blurring of tissue types, particularly in white matter. Our results indicated that this was indeed the case. More specifically, we noticed in regions of high anisotropy (WM) that anisotropic kernel (AG) smoothing reduced the partial volume averaging between tissue types. However, in low anisotropy region (GM) isotropic Gaussian filter performed best. Perona Malik algorithm was good at boundary regions. Overall, these results suggest that the optimum filter properties depend upon the type of tissue being investigated.

As discussed in the Introduction, several anisotropic filtering algorithms have been developed to regularize and denoise DTI data. These algorithms have focused on the preservation of discontinuities in the raw image data. In many of these previous filtering studies, the apparent objective has been to regularize the estimates of the white matter tract directions (e.g., Tschumperle et al. [7]; Coulon et al. [2]; Wang et al. [8]) in noisy tensor field data. This is important for white matter tractography, but less so for quantitative DTI studies. In our study, the effects of spatial filtering methods on the quantification of important DTI measures were evaluated. Future studies are necessary to evaluate and compare other anisotropic spatial filtering and regularization methods for DTI data.

\section{REFERENCES}

[1] Chung, M.K., Lazar, M., Alexander, AL., Lu, Y., and Davidson, R., " Probabilistic Connectivity Measure in Diffusion Tensor Imaging via Anisotropic Kernel Smoothing," UW Dept of Stats Technical Report NO. 1081, 2003.

[2] Coulon, O., Alexander, D.C., and Arridge, S., "Diffusion tensor magnetic resonance image regularization," Medical image analysis, 8, pp. 47-67, 2004.

[3] Pajevic, S., Aldroubi, A., and Basser, PJ., "A continuous tensor field approximation of discrete DT-MRI data for extracting Microstructural and architectural features of tissues," $J$ Magn Reson, 154, pp. 85-100, 2002.

[4] Parker, G.J.M., Schnabel, J.A., Symms, M.R., Werring, D.J., and Barker, G.J., "Nonlinear Smoothing for Reduction of Systematic and Random Errors in Diffusion Tensor Imaging," JMRI, vol. 11, pp. 702-710, 2000.

[5] Perona, P., and Malik, J., "Scale-space and edge detection using anisotropic diffusion," IEEE Trans. Pattern Anal. Machine Intell, vol. 12, pp. 629-639, 1990.

[6] Pierpaoli, C., and Basser, PJ., "Toward a quantitative assessment of diffusion anisotropy," Magn. Reson. Med, vol. 36, pp. 893-906, 1996

[7] Tschumperle, D., and Deriche, R., "DT-MRI: Estimation, Regularization and Applications," Eurocast $9^{\text {th }}$ international conference on computer aided systems theory, 2003.

[8] Wang, Z., Vemuri, B.C., Chen, Y., and Mareci, T.H., "A Constrained Variational for Direct Estimation and Smoothing of the Diffusion Tensor Field From complex DWI," IEEE

Transactions on medical imaging, vol. 23, pp. 930-939, 2004. 